\title{
Imaginary quadratic fields whose Iwasawa $\lambda$-invariant is equal to 1
}

\author{
by \\ Dongho Byeon (Seoul)
}

1. Introduction and statement of results. Let $D$ be the fundamental discriminant of the quadratic field $\mathbb{Q}(\sqrt{D})$ and $\chi_{D}:=\left(\frac{D}{n}\right)$ the usual Kronecker character. Let $p$ be a prime, $\mathbb{Z}_{p}$ the ring of $p$-adic integers, and $\lambda_{p}(\mathbb{Q}(\sqrt{D}))$ the Iwasawa $\lambda$-invariant of the cyclotomic $\mathbb{Z}_{p}$-extension of $\mathbb{Q}(\sqrt{D})$. In this paper, we shall prove the following:

Theorem 1.1. For any odd prime $p$,

$$
\sharp\left\{-X<D<0 \mid \lambda_{p}(\mathbb{Q}(\sqrt{D}))=1, \chi_{D}(p)=1\right\} \gg \frac{\sqrt{X}}{\log X} .
$$

Horie [9] proved that for any odd prime $p$, there exist infinitely many imaginary quadratic fields $\mathbb{Q}(\sqrt{D})$ with $\lambda_{p}(\mathbb{Q}(\sqrt{D}))=0$, and the author [1] gave a lower bound for the number of such imaginary quadratic fields. It is known that for any prime $p$ which splits in the imaginary quadratic field $\mathbb{Q}(\sqrt{D}), \lambda_{p}(\mathbb{Q}(\sqrt{D})) \geq 1$. So it is interesting to see how often the trivial $\lambda$-invariant appears for such a prime. Jochnowitz [10] proved that for any odd prime $p$, if there exists one imaginary quadratic field $\mathbb{Q}\left(\sqrt{D_{0}}\right)$ with $\lambda_{p}\left(\mathbb{Q}\left(\sqrt{D_{0}}\right)\right)=1$ and $\chi_{D_{0}}(p)=1$, then there exist an infinite number of such imaginary quadratic fields.

For the case of real quadratic fields, Greenberg [8] conjectured that $\lambda_{p}(\mathbb{Q}(\sqrt{D}))=0$ for all real quadratic fields and all prime numbers $p$. Ono [11] and Byeon [2], [3] showed that for all prime numbers $p$, there exist infinitely many real quadratic fields $\mathbb{Q}(\sqrt{D})$ with $\lambda_{p}(\mathbb{Q}(\sqrt{D}))=0$ and gave a lower bound for the number of such real quadratic fields.

In Section 3, we shall prove the following:

2000 Mathematics Subject Classification: 11R11, 11R23.

This work was supported by grant no. R08-2003-000-10243-0 from the Basic Research Program of the Korea Science and Engineering Foundation. 
Proposition 1.2. For any odd prime $p$, if there is a negative fundamental discriminant $D_{0}<0$ such that $\lambda_{p}\left(\mathbb{Q}\left(\sqrt{D_{0}}\right)\right)=1$ and $\chi_{D_{0}}(p)=1$, then

$$
\sharp\left\{-X<D<0 \mid \lambda_{p}(\mathbb{Q}(\sqrt{D}))=1, \chi_{D}(p)=1\right\} \gg \frac{\sqrt{X}}{\log X} .
$$

In Section 4, we shall prove the following:

Proposition 1.3. Let $p$ be an odd prime and $D_{0}<0$ be the fundamental discriminant of the imaginary quadratic field $\mathbb{Q}\left(\sqrt{1-p^{2}}\right)$. Then $\chi_{D_{0}}(p)=1$ and $\lambda_{p}\left(\mathbb{Q}\left(\sqrt{D_{0}}\right)\right)=1$ if and only if $2^{p-1} \not \equiv 1\left(\bmod p^{2}\right)$, that is, $p$ is not a Wieferich prime.

Proposition 1.4. Let $p$ be a Wieferich prime. If $p \equiv 3(\bmod 4)$, let $D_{0}<0$ be the fundamental discriminant of the imaginary quadratic field $\mathbb{Q}(\sqrt{1-p})$, and if $p \equiv 1(\bmod 4)$, let $D_{0}<0$ be the fundamental discriminant of the imaginary quadratic field $\mathbb{Q}(\sqrt{4-p})$. Then $\chi_{D_{0}}(p)=1$ and $\lambda_{p}\left(\mathbb{Q}\left(\sqrt{D_{0}}\right)\right)=1$.

From these three propositions, Theorem 1.1 follows.

2. Preliminaries. Let $\chi$ be a non-trivial even primitive Dirichlet character of conductor $f$ which is not divisible by $p^{2}$. Let $L_{p}(s, \chi)$ be the KubotaLeopoldt $p$-adic $L$-function and $O_{\chi}=\mathbb{Z}_{p}[\chi(1), \chi(2), \ldots]$. Then there is a power series $F(T, \chi) \in O_{\chi}[[T]]$ such that

$$
L_{p}(s, \chi)=F\left((1+p d)^{s}-1, \chi\right),
$$

where $d=f$ if $p \nmid f$ and $d=f / p$ if $p \mid f$. Let $\pi$ be a generator for the ideal of $O_{\chi}$ above $p$. Then we may write

$$
F(T, \chi)=G(T) U(T),
$$

where $U(T)$ is a unit of $O_{\chi}[[T]]$, and $G(T)$ is a distinguished polynomial: that is, $G(T)=a_{0}+a_{1} T+\cdots+T^{\lambda}$ with $\pi \mid a_{i}$ for $i \leq \lambda-1$. Define $\lambda\left(L_{p}(s, \chi)\right)$ to be the index of the first coefficient of $F(T, \chi)$ not divisible by $\pi$. Let $\omega$ be the Teichmüller character.

Lemma 2.1 (Dummit, Ford, Kisilevsky and Sands [6, Proposition 5.1]). Let $D<0$ be the fundamental discriminant of the imaginary quadratic field $\mathbb{Q}(\sqrt{D})$. Then

$$
\lambda_{p}(\mathbb{Q}(\sqrt{D}))=\lambda\left(L_{p}\left(s, \chi_{D} \omega\right)\right) .
$$

Lemma 2.2 (Washington [13, Lemma 1]). Let $D<0$ be the fundamental discriminant of the imaginary quadratic field $\mathbb{Q}(\sqrt{D})$. Then

$$
\lambda\left(L_{p}\left(s, \chi_{D} \omega\right)\right)=1 \Leftrightarrow L_{p}\left(0, \chi_{D} \omega\right) \not \equiv L_{p}\left(1, \chi_{D} \omega\right)\left(\bmod p^{2}\right) .
$$

From these lemmas, we can show the following: 
Proposition 2.3. Let $p$ be an odd prime and $D<0$ be the fundamental discriminant of the imaginary quadratic field $\mathbb{Q}(\sqrt{D})$ such that $\chi_{D}(p)=1$. Then $L\left(1-p, \chi_{D}\right) / p$ is p-integral and

$$
\lambda_{p}(\mathbb{Q}(\sqrt{D}))=1 \Leftrightarrow \frac{L\left(1-p, \chi_{D}\right)}{p} \not \equiv 0(\bmod p),
$$

where $L\left(s, \chi_{D}\right)$ is the Dirichlet L-function.

Proof. By the construction of the $p$-adic $L$-function $L_{p}\left(s, \chi_{D}\right)$,

$$
L_{p}\left(0, \chi_{D} \omega\right)=-\left(1-\chi_{D} \omega \cdot \omega^{-1}(p)\right) B_{1, \chi_{D} \omega \cdot \omega^{-1}}=-\left(1-\chi_{D}(p)\right) B_{1, \chi_{D}},
$$

where $B_{n, \chi_{D}}$ is the generalized Bernoulli number. Since $\chi_{D}(p)=1$,

$$
L_{p}\left(0, \chi_{D} \omega\right)=0 .
$$

Similarly,

$$
\begin{aligned}
L_{p}\left(1-p, \chi_{D} \omega\right) & =-\left(1-\chi_{D} \omega \cdot \omega^{-p}(p) p^{p-1}\right) B_{p, \chi_{D} \omega \cdot \omega^{-p}} / p \\
& =-\left(1-\chi_{D}(p) p^{p-1}\right) B_{p, \chi_{D}} / p=\left(1-p^{p-1}\right) L\left(1-p, \chi_{D}\right) \\
& \equiv L\left(1-p, \chi_{D}\right)\left(\bmod p^{2}\right) .
\end{aligned}
$$

Since $\chi_{D} \omega \neq 1$ is not a character of the second kind, $L_{p}\left(1-p, \chi_{D} \omega\right)$ and $L\left(1-p, \chi_{D}\right)$ are $p$-integral (see [14]). By the congruence of $L_{p}\left(s, \chi_{D}\right)$,

$$
L_{p}\left(1, \chi_{D} \omega\right) \equiv L_{p}\left(0, \chi_{D} \omega\right)=0(\bmod p)
$$

and

$$
L_{p}\left(1, \chi_{D} \omega\right) \equiv L_{p}\left(1-p, \chi_{D} \omega\right)\left(\bmod p^{2}\right) .
$$

Thus $L\left(1-p, \chi_{D}\right) / p$ is $p$-integral and

$$
\frac{L\left(1-p, \chi_{D}\right)}{p} \not \equiv 0(\bmod p) \Leftrightarrow L_{p}\left(1, \chi_{D} \omega\right) \not \equiv 0\left(\bmod p^{2}\right) .
$$

From (1) and Lemmas 2.1, 2.2, the proposition follows.

3. Proof of Proposition 1.2. Let $M_{k}\left(\Gamma_{0}(N), \chi\right)$ denote the space of modular forms of weight $k$ on $\Gamma_{0}(N)$ with character $\chi$. For a positive integer $r \geq 2$, let

$$
F_{r}(z):=\sum_{N \neq 0} H(r, N) q^{N} \in M_{r+1 / 2}\left(\Gamma_{0}(4), \chi_{0}\right)
$$

be the Cohen modular form [4], where $q:=e^{2 \pi i z}$. We note that if $D n^{2}=$ $(-1)^{r} N$, then

$$
H(r, N)=L\left(1-r, \chi_{D}\right) \sum_{d \mid n} \mu(d) \chi_{D}(d) d^{r-1} \sigma_{2 r-1}(n / d),
$$


where $\sigma_{\nu}(n):=\sum_{d \mid n} d^{\nu}$. From $F_{p}(z)$, we can construct the modular form

$$
G_{p}(z):=\sum_{\left(\frac{-n}{p}\right)=1,\left(\frac{n}{Q}\right)=-1} \frac{H(p, n)}{p} q^{n} \in M_{p+1 / 2}\left(\Gamma_{0}\left(4 p^{4} Q^{4}\right), \chi_{0}\right),
$$

where $Q$ is a prime such that $Q \neq p$. From Proposition 2.3 and equation (2), if $D<0$ is the fundamental discriminant of the imaginary quadratic field $\mathbb{Q}(\sqrt{D})$ such that $\chi_{D}(p)=1$, then

$$
\frac{H(p,-D)}{p}=\frac{L\left(1-p, \chi_{D}\right)}{p}
$$

is $p$-integral. Using similar methods to Ono [11] and Byeon [2], that is, applying a theorem of Sturm [12] to the following two modular forms:

$$
\begin{aligned}
& \left(U_{l} \mid G_{p}\right)(z)=\sum_{\left(\frac{-n}{p}\right)=1,\left(\frac{n}{Q}\right)=-1} \frac{H(p, l n)}{p} q^{n} \in M_{p+1 / 2}\left(\Gamma_{0}\left(4 p^{4} Q^{4} l\right),\left(\frac{4 l}{\cdot}\right)\right), \\
& \left(V_{l} \mid G_{p}\right)(z)=\sum_{\left(\frac{-n}{p}\right)=1,\left(\frac{n}{Q}\right)=-1} \frac{H(p, n)}{p} q^{l n} \in M_{p+1 / 2}\left(\Gamma_{0}\left(4 p^{4} Q^{4} l\right),\left(\frac{4 l}{\cdot}\right)\right),
\end{aligned}
$$

where $l \neq p$ is a suitable prime, and comparing the coefficients of $q^{-D_{0} l^{3}}$ in these modular forms, where $D_{0}<0$ is a fundamental discriminant of the imaginary quadratic field $\mathbb{Q}\left(\sqrt{D_{0}}\right)$ such that $\chi_{D_{0}}(p)=1$ and $H\left(p,-D_{0}\right) / p \not \equiv$ $0(\bmod p)$, we can obtain the following:

Proposition 3.1. Let $p$ be an odd prime. Assume that there is a fundamental discriminant $D_{0}<0$ of the imaginary quadratic field $\mathbb{Q}\left(\sqrt{D_{0}}\right)$ such that

(i) $\chi_{D_{0}}(p)=1$,

(ii) $H\left(p,-D_{0}\right) / p \not \equiv 0(\bmod p)$.

Then there is an arithmetic progression $r_{p}\left(\bmod p t_{p}\right)$ with $\left(r_{p}, p t_{p}\right)=1$ and $\left(\frac{-r_{p}}{p}\right)=1$, and a constant $\kappa(p)$ such that for each prime $l \equiv r_{p}\left(\bmod p t_{p}\right)$ there is an integer $1 \leq d_{l} \leq \kappa(p) l$ for which

(i) $D_{l}:=-d_{l} l$ is a fundamental discriminant,

(ii) $H\left(p,-D_{l}\right) / p \not \equiv 0(\bmod p)$.

Proof of Proposition 1.2. Let $D_{l}<0$ be the fundamental discriminant in Proposition 3.1. Then $\chi_{D_{l}}(p)=1$ and $H\left(p,-D_{l}\right) / p=L\left(1-p, \chi_{D_{l}}\right) / p \not \equiv$ $0(\bmod p)$. By Proposition 2.3, $\lambda_{p}\left(\mathbb{Q}\left(\sqrt{D_{l}}\right)\right)=1$. By Dirichlet's theorem on primes in arithmetic progression, the number of such $D_{l}<X$ is $\gg$ $\sqrt{X} / \log X$. 
4. Proof of Propositions 1.3 and 1.4. To prove these propositions, we shall use the following criterion of Gold.

Lemma 4.1 (Gold [7]). Let $p$ be an odd prime and $D<0$ be the fundamental discriminant of the imaginary quadratic field $\mathbb{Q}(\sqrt{D})$ such that $\chi_{D}(p)=1$. Let $(p)=\mathbf{P} \overline{\mathbf{P}}$ in $\mathbb{Q}(\sqrt{D})$. Suppose that $\mathbf{P}^{r}=(\pi)$ is principal for some integer $r$ not divisible by $p$. Then $\lambda_{p}(\mathbb{Q}(\sqrt{D}))=1$ if and only if $\pi^{p-1} \not \equiv 1\left(\bmod \overline{\mathbf{P}}^{2}\right)$.

Proof of Proposition 1.3. We note that $1-p^{2}$ is not a square. Let $\mathbf{P}=$ $\left(p, 1+\sqrt{1-p^{2}}\right)$ and $\overline{\mathbf{P}}=\left(p, 1-\sqrt{1-p^{2}}\right)$. Then $(p)=\mathbf{P} \overline{\mathbf{P}}$ and $\mathbf{P}^{2}=$ $\left(1+\sqrt{1-p^{2}}\right), \overline{\mathbf{P}}^{2}=\left(1-\sqrt{1-p^{2}}\right)$. From Lemma $4.1, \lambda_{p}\left(\mathbb{Q}\left(\sqrt{D_{0}}\right)\right)=1$ if and only if

$$
\left(1+\sqrt{1-p^{2}}\right)^{p-1} \not \equiv 1\left(\bmod 1-\sqrt{1-p^{2}}\right) .
$$

This is equivalent to

$$
\begin{aligned}
\left(1+\sqrt{1-p^{2}}\right)^{p}-\left(1+\sqrt{1-p^{2}}\right) & \neq 0 \\
\left(\bmod p^{2}\right. & \left.=\left(1-\sqrt{1-p^{2}}\right)\left(1+\sqrt{1-p^{2}}\right)\right) .
\end{aligned}
$$

We see that

$$
\begin{aligned}
(1+ & \left.\sqrt{1-p^{2}}\right)^{p}-\left(1+\sqrt{1-p^{2}}\right) \\
& \equiv \sum_{n=0}^{(p-1) / 2}\left(\begin{array}{c}
p \\
2 n
\end{array}\right)+\left(\sum_{n=0}^{(p-1) / 2}\left(\begin{array}{c}
p \\
2 n+1
\end{array}\right)\right) \sqrt{1-p^{2}}-\left(1+\sqrt{1-p^{2}}\right) \\
& \equiv\left(\sum_{n=0}^{(p-1) / 2}\left(\begin{array}{c}
p \\
2 n
\end{array}\right)-1\right)+\left(\sum_{n=0}^{(p-1) / 2}\left(\begin{array}{c}
p \\
2 n+1
\end{array}\right)-1\right) \sqrt{1-p^{2}} \\
& \equiv\left(2^{p-1}-1\right)\left(1+\sqrt{1-p^{2}}\right)\left(\bmod p^{2}\right),
\end{aligned}
$$

where we have used the fact that

$$
\sum_{n=0}^{(p-1) / 2}\left(\begin{array}{c}
p \\
2 n
\end{array}\right)=\sum_{n=0}^{(p-1) / 2}\left(\begin{array}{c}
p \\
2 n+1
\end{array}\right)=2^{p-1}
$$

Thus $(3)$ is true if and only if $2^{p-1} \not \equiv 1\left(\bmod p^{2}\right)$, that is, $p$ is not a Wieferich prime, and the proposition follows.

Proof of Proposition 1.4. We note that $1-p$ is not a square if $p \equiv 3$ $(\bmod 4)$ and $4-p$ is not a square if $p \equiv 1(\bmod 4)$. We also note that $\chi_{D_{0}}(p)=1$. First we consider the case $p \equiv 3(\bmod 4)$. Let $\mathbf{P}=(1+\sqrt{1-p})$ and $\overline{\mathbf{P}}=(1-\sqrt{1-p})$. Then $(p)=\mathbf{P} \overline{\mathbf{P}}$ and $\mathbf{P}^{2}=\left((1+\sqrt{1-p})^{2}\right), \overline{\mathbf{P}}^{2}=$ $\left((1-\sqrt{1-p})^{2}\right)$. Now, from Lemma $4.1, \lambda_{p}\left(\mathbb{Q}\left(\sqrt{D_{0}}\right)\right)=1$ if and only if

$$
(1+\sqrt{1-p})^{2(p-1)} \not \equiv 1\left(\bmod (1-\sqrt{1-p})^{2}\right) .
$$


This is equivalent to

$$
\begin{aligned}
(1+\sqrt{1-p})^{2 p}-(1+\sqrt{1-p})^{2} & \neq 0 \\
\left(\bmod p^{2}\right. & \left.=(1-\sqrt{1-p})^{2}(1+\sqrt{1-p})^{2}\right) .
\end{aligned}
$$

We see that

$$
\begin{aligned}
(1+ & \sqrt{1-p})^{2 p} \\
\equiv & \sum_{n=0}^{p}\left(\left(\begin{array}{c}
2 p \\
2 n
\end{array}\right)(1-p)^{n}\right)+\sqrt{1-p} \cdot \sum_{n=0}^{p-1}\left(\left(\begin{array}{c}
2 p \\
2 n+1
\end{array}\right)(1-p)^{n}\right) \\
\equiv & \sum_{n=0}^{p}\left(\left(\begin{array}{c}
2 p \\
2 n
\end{array}\right)(1-n p)\right)+\sqrt{1-p} \cdot \sum_{n=0}^{p-1}\left(\left(\begin{array}{c}
2 p \\
2 n+1
\end{array}\right)(1-n p)\right) \\
\equiv & \sum_{n=0}^{p}\left(\begin{array}{c}
2 p \\
2 n
\end{array}\right)-p \cdot \sum_{n=0}^{p} n\left(\begin{array}{c}
2 p \\
2 n
\end{array}\right) \\
& +\sqrt{1-p} \cdot\left(\sum_{n=0}^{p-1}\left(\begin{array}{c}
2 p \\
2 n+1
\end{array}\right)-p \cdot \sum_{n=0}^{p-1} n\left(\begin{array}{c}
2 p \\
2 n+1
\end{array}\right)\right)\left(\bmod p^{2}\right),
\end{aligned}
$$

where we have used the fact that $(1-p)^{n} \equiv 1-n p\left(\bmod p^{2}\right)$. Now, since

$$
\begin{aligned}
& \sum_{n=0}^{p}\left(\begin{array}{c}
2 p \\
2 n
\end{array}\right)=\sum_{n=0}^{p-1}\left(\begin{array}{c}
2 p \\
2 n+1
\end{array}\right)=2^{2 p-1}, \\
& \sum_{n=1}^{p} n\left(\begin{array}{c}
2 p \\
2 n
\end{array}\right)=p \cdot 2^{2 p-2} \\
& \sum_{n=1}^{p-1} n\left(\begin{array}{c}
2 p \\
2 n+1
\end{array}\right)=(p-1) \cdot 2^{2 p-2}
\end{aligned}
$$

we find that

$$
(1+\sqrt{1-p})^{2 p} \equiv 2^{2 p-1}+\sqrt{1-p} \cdot\left(2^{2 p-1}+p \cdot 2^{2 p-2}\right)\left(\bmod p^{2}\right) .
$$

Hence

$$
\begin{aligned}
& (1+\sqrt{1-p})^{2 p}-(1+\sqrt{1-p})^{2} \\
& \quad \equiv\left(2^{2 p-1}+p-2\right)+\left(2^{2 p-1}+p \cdot 2^{2 p-2}-2\right) \sqrt{1-p}\left(\bmod p^{2}\right)
\end{aligned}
$$

Thus (4) is true if and only if

(5) $2^{2 p-1}+p-2 \not \equiv 0\left(\bmod p^{2}\right) \quad$ or $2^{2 p-1}+p \cdot 2^{2 p-2}-2 \not \equiv 0\left(\bmod p^{2}\right)$.

But it is easy to see that $(5)$ is true if $2^{p-1} \equiv 1\left(\bmod p^{2}\right)$. Hence if $p$ is a Wieferich prime, then $\lambda_{p}\left(\mathbb{Q}\left(\sqrt{D_{0}}\right)\right)$ should be equal to 1 .

Now we consider the case $p \equiv 1(\bmod 4)$. Let $\mathbf{P}=(2+\sqrt{4-p})$ and $\overline{\mathbf{P}}=(2-\sqrt{4-p})$. Then $(p)=\mathbf{P} \overline{\mathbf{P}}$ and $\mathbf{P}^{2}=\left((2+\sqrt{4-p})^{2}\right), \overline{\mathbf{P}}^{2}=$ 
$\left((2-\sqrt{4-p})^{2}\right)$. Then from Lemma $4.1, \lambda_{p}\left(\mathbb{Q}\left(\sqrt{D_{0}}\right)\right)=1$ if and only if

$$
(2+\sqrt{4-p})^{2(p-1)} \not \equiv 1\left(\bmod (2-\sqrt{4-p})^{2}\right) .
$$

This is equivalent to

$$
\begin{aligned}
(2+\sqrt{4-p})^{2 p}-(2+\sqrt{4-p})^{2} & \neq 0 \\
\left(\bmod p^{2}\right. & \left.=(2-\sqrt{4-p})^{2}(2+\sqrt{4-p})^{2}\right) .
\end{aligned}
$$

By a computation similar to the above, we have

$$
\begin{aligned}
& (2+\sqrt{4-p})^{2 p}-(2+\sqrt{4-p})^{2} \\
& \quad \equiv\left(2^{4 p-1}+p-8\right)+\left(2^{4 p-2}+p \cdot 2^{4 p-5}-4\right) \sqrt{4-p}\left(\bmod p^{2}\right) .
\end{aligned}
$$

Thus (6) is true if and only if

$$
2^{4 p-1}+p-8 \not \equiv 0\left(\bmod p^{2}\right) \quad \text { or } \quad 2^{4 p-2}+p \cdot 2^{4 p-5}-4 \not \equiv 0\left(\bmod p^{2}\right) .
$$

But it is also easy to see that $(7)$ is true if $2^{p-1} \equiv 1\left(\bmod p^{2}\right)$. Hence if $p$ is a Wieferich prime, then $\lambda_{p}\left(\mathbb{Q}\left(\sqrt{D_{0}}\right)\right)$ should be equal to 1 , and we have proved the proposition.

REMARK. It seems interesting that Propositions 1.3 and 1.4 give criteria for the Wieferich primes. We know that the Wieferich primes are very rare. The only Wieferich primes for $p \leq 4 \cdot 10^{12}$ are $p=1093$ and $p=3511$ (see [5]).

Acknowledgements. The author thanks the referee for many valuable suggestions and for correcting several mistakes.

\section{References}

[1] D. Byeon, A note on basic Iwasawa $\lambda$-invariants of imaginary quadratic fields and congruence of modular forms, Acta Arith. 89 (1999), 295-299.

[2] - Indivisibility of class numbers and Iwasawa $\lambda$-invariants of real quadratic fields, Compositio Math. 126 (2001), 249-256.

[3] - Existence of certain fundamental discriminants and class numbers of real quadratic fields, J. Number Theory 98 (2003), 432-437.

[4] H. Cohen, Sums involving the values at negative integers of L-functions of quadratic characters, Math. Ann. 217 (1975), 271-285.

[5] R. Crandall, K. Dilcher and C. Pomerance, A search for Wieferich and Wilson primes, Math. Comp. 66 (1997), 433-449.

[6] D. S. Dummit, D. Ford, H. Kisilevsky and J. W. Sands, Computation of Iwasawa lambda invariants for imaginary quadratic fields, J. Number Theory 37 (1991), $100-121$.

[7] R. Gold, The nontriviality of certain $\mathbb{Z}_{l}$-extensions, ibid. 6 (1974), 369-373.

[8] R. Greenberg, On the Iwasawa invariants of totally real number fields, Amer. J. Math. 98 (1976), 263-284.

[9] K. Horie, A note on basic Iwasawa $\lambda$-invariants of imaginary quadratic fields, Invent. Math. 88 (1987), 31-38. 
[10] N. Jochnowitz, A p-adic conjecture about derivatives of L-series attached to modular forms, in: p-adic Monodromy and the Birch and Swinnerton-Dyer Conjecture (Boston, MA, 1991), Contemp. Math. 165, Amer. Math. Soc., Providence, RI, 1994, 239-263.

[11] K. Ono, Indivisibility of class numbers of real quadratic fields, Compositio Math. 119 (1999), 1-11.

[12] J. Sturm, On the congruence of modular forms, in: Number Theory (New York, 1984-1985), Lecture Notes in Math. 1240, Springer, Berlin, 1984, 275-280.

[13] L. C. Washington, Zeros of p-adic L-functions, in: Séminaire Delange-Pisot-Poitou (Séminaire de Théorie des Nombres, Paris, 1980/1981), Progr. Math. 22, Birkhäuser, Boston, 1982, 337-357.

[14] - Introduction to Cyclotomic Fields, Grad. Texts in Math. 83, Springer, New York, 1997.

Department of Mathematics

Seoul National University

Seoul 151-747, Korea

E-mail: dhbyeon@math.snu.ac.kr

Received on 12.10.2004

and in revised form on 7.7.2005 